Article

\title{
Comparisons of Pre-Sale Strategies for a Fresh Agri-Product Supply Chain with Service Effort
}

\author{
Huanyong Zhang, Huiyuan $\mathrm{Xu}$ * and Xujin $\mathrm{Pu}$ \\ Business School, Jiangnan University, Wuxi 214122, China; zhanghy@jiangnan.edu.cn (H.Z.); \\ puyiwei@ustc.edu (X.P.) \\ * Correspondence: 6180905010@stu.jiangnan.edu.cn; Tel.:+86-150-6188-3764
}

Received: 6 July 2020; Accepted: 28 July 2020; Published: 2 August 2020

\begin{abstract}
Preselling strategies have been a common marketing tool, but research on this selection is limited. Thus, we examine three pre-sale strategies of a manufacturer who produces and sells a seasonal product to a retailer: (1) supplier pre-sale strategy-the supplier carries out preselling by opening a direct channel; (2) retailer pre-sale strategy-the retailer purchases pre-sale products from the supplier and sells them in online and offline channels; and (3) joint pre-sale strategy-the retailer acts as a pre-sale platform, providing order information and pre-sale services. For each preselling mode, we construct the Stackelberg game model aiming to maximize profits and obtain optimal service levels and pricing decisions. We find that the two latter scenarios, that is, to cooperate with the retailer (retailer pre-sale and joint pre-sale strategies), could highly gain more profit for the supplier compared with the former. For the supplier, the joint pre-sale strategy will likely be the dominant strategy because of its wide-range applicability. On the contrary, the retailer is swaying between retailer pre-sale and joint pre-sale strategies under different conditions. For the supplier, leadership always guarantees a high profit, but if the joint pre-sale strategy is adopted, then the existence of a profit-sharing ratio will narrow the profit gap between the two players.
\end{abstract}

Keywords: pre-sale strategy; service level; supply chain management; game theory

\section{Introduction}

Currently, the sale process of some products is divided into two stages, namely, the pre-sale and spot sale stages. For instance, Huawei allowed consumers to pre-order when unveiling its latest products, and some suppliers also presell fresh agri-products to potential consumers. The pre-sale strategy is common in various industries [1]. Numerous studies on preselling have been conducted, and results show that preselling provides many benefits for the entire supply chain. Initially, preselling can support the demand forecasts in the later periods [2], reducing the retailer's ordering and production risks. Second, the pre-sale strategy provides leverage for the retailer to charge different prices at different sale stages [3]. During the pre-sale stage, offering a discount simulates consumer purchases, and the increasing demand improves the profit of the retailer and supplier. Additionally, the positive effect of product diffusion leads to a strong demand arrival going forward potentially in the later periods $[4,5]$. In terms of the profit performance of the pre-sale strategy, as previously reported, the one-hour pre-sale performance of the 2020 Tmall 618 shopping festival increased by 515\% year-on-year. Therefore, preselling becomes a strategic tool to compete for customers to obtain the best performance [6]. Since pre-sales brings minions of benefits, the problem arises: how will a company planning to carry out pre-sales determine its pre-sale strategy? However, this problem has drawn little attention, and the mechanism behind the pre-sale strategy selection remains unclear. Therefore, given the popularity of the pre-sale strategy, it is practically meaningful to investigate the pre-sale strategy from the perspective of supply chain management. 
Preselling and service are a form of separating purchase from consumption. Thus, consumers are uncertain about their utility until they perceive it, resulting in some consumers considering not only pre-sale and spot prices but also concerns about service levels. Additionally, many companies apply service differentiation strategies as another critical competitive strategy due to the prevalence of consumer-centric business philosophy. Hence, providing good service is also an important priority when applying pre-sale strategy in a fresh agri-product supply chain. We integrate service decisions into the supply chain to explore the relationship between price and service level decisions.

We also provide a brief review of the literature relevant to our research. Preselling may have been first introduced in the retail industry by Fisher and Raman [7]. In the early days of applying the pre-sale strategy, transaction costs and arbitrage prevented some companies from benefiting as most companies do. However, new technologies are overcoming the two impediments to preselling, enabling advanced selling in numerous industries [8]. In recent years, preselling plays various roles in improving business operations and has received a considerable amount of attention. We can broadly categorize them into three groups. The first group focuses on the demand forecast benefit of advance selling and shows that advance selling can help firms obtain advance demand information and thus improve the precision of demand forecasts. Among these, Kevin Weng and Parlar [9] may be the first to integrate advance selling with supply- and demand-side elements, aiming to find the optimal inventory and discount level. Similarly, Boyac and Özer [10] identified the conditions under which advance selling for capacity planning is the most valuable and found that advance selling can significantly increase the manufacturer's profit. The second line of literature integrates the problem of resource constraints with the preselling strategy. For instance, Guo [11] considered advance selling in an oligopolistic market with capacity constraints. Xiao and Zhang [12] and Jin et al. [13] utilized the pre-sale strategy as a financing strategy for a capital-constrained supply chain. Some literature considers consumers' behaviors with pre-sale strategies into a supply chain, such as consumer loss aversion [14], forward-looking consumers [15], and strategic consumers [16]. Furthermore, some focus on preselling as a vital tool to compete for customers [5,8], which is also the basis of our study.

Besides, numerous studies regard service decisions in operations management, and many of them concern service and pricing strategies [17-19]. Yu and Xiao [18] investigated the pricing and service level decisions of a fresh agri-product supply chain. Our study is similar but with some key differences. In our study, we consider the pricing and service-level decisions in a supply chain integrating pre-sales and spot sales, and we concentrate on the pre-sale strategy selection. Jin et al. [20] focused on the buy-online-pick-up-in-store mode in an omnichannel retailing environment, which is similar to the service provided in our model. When the supplier or retailer adopts a preselling strategy, pre-ordering online and picking up in-store or delivery to the home is a standard pre-sale method. However, the literature for integrating service with an advanced selling strategy is limited. Shugan and Xie [8] discussed a scenario where advanced selling strategies are applied by service providers and emphasized the advantages of advance selling by comparing them with spot sales. The above literature analysis shows the fact that few studies investigate service and pricing decisions with pre-sale strategies. Unlike them, our concern is the optimal pre-sale strategy selection. We contribute to the existing works in a supply chain integrated pre-sale and spot sale by exploring the service and pricing decision and optimal profit of the supply player.

This study investigates the optimal pre-sale strategy for a supplier in a supply chain integrated spot sale and pre-sale strategies. We individually consider a fresh agri-product supply chain comprising a dominant supplier and a retailer with online and offline channels. Our primary research questions are as follows: Which model is suitable for the supplier to carry out pre-sale independently or to cooperate with the retailer? If collaborating with the retailer gains additional money, will the supplier prefer the retailer pre-sale strategy or joint pre-sale one? How do the price and service level vary under different pre-sale policies? How do key parameters influence the optimal pre-sale strategy choices?

By resolving the above questions, we try to discover the principles behind the selection of pre-sale strategies and the optimal supply chain decisions of participants. Specifically, we use analytical and 
numerical means to better understand the conditions under which pre-sale strategies are the most profitable. In general, this study could enrich the supply chain operation management considering preselling, and it can also provide some operational references for companies that are about to adopt pre-sale strategies. Comparing the optimal pricing, service level, and profit under the three pre-sale strategies, the pre-sales strategy preference of supply chain members are obtained. The results show that the supplier prefers to cooperate with the retailer to pre-sell the product to them. Cooperation usually brings additional profit for the supplier. Specifically, the joint pre-sale strategy will be the first choice of the supplier given its wide-range applicability, whereas the retailer is swaying between retailer pre-sale and joint pre-sale strategies under different conditions. Leadership always guarantees the supplier a high profit. However, if the joint pre-sale strategy is adopted, then the existence of the profit-sharing ratio will narrow the profit gap between the two players, which is a significant advantage over the other models.

\section{Materials and Methods}

\subsection{Model Specifications}

We consider a fresh product supply chain consisting of one supplier and one retailer. The supplier produces products and sells them to the end customers via the retailer's online and offline stores. Attracted by the advantages of the pre-sale strategy we have mentioned above, the supplier now desires to apply a pre-sale plan to increase profits. The supplier also has concerns about the level of pre-sale services, such as cold-chain and delivery services. We assume that the supplier can choose from three pre-sale strategies. The first is the supplier pre-sale, in which the supplier carries out the preselling himself. We assume that the supplier had no direct channel before, so opening a direct channel is necessary to presell and provide pre-sale services to consumers. Specifically, the supplier needs to afford the cost of pre-sale services $C_{s}$ and start-up capital $a$. We assume that the cost of the pre-sale service is related to the service level, $C_{s}=\frac{m s_{s}^{s_{s}^{2}}}{2}$, where $m>0$. The convex quadratic function is a common assumption, as shown in Xiao and Yang [21] and Pi, Fang, and Zhang [17]. The second one is the retailer pre-sales strategy. Under this strategy, the retailer purchases pre-sale and spot products from the supplier at $w_{1}, w_{2}$ and sells them in online and offline stores at $p_{1}, p_{2}$, respectively. The last one is the joint pre-sale strategy. The retailer acts as a pre-sale platform and provides ordering information and pre-sale services. At the time, the supplier presells products to consumers, and the retailer offers pre-sale service to consumers. Similarly, the retailer faces the cost of pre-sale service at $C_{r}=\frac{n s_{r}^{s 2}}{2}$, where $n>0$. Then, we describe the three pre-sale strategies in Figure 1.

In fresh agri-product supply chains, demand functions in the pre-sale stage and spot sale stage with respect to the service level and the sales price are commonly used, as shown in Wu et al. [22] and Yu and Xiao [18]. Market demand is positively influenced by service levels but negatively impacted by increased retail prices. Under three pre-sale strategies, market demand from the supplier's channel during the pre-sale stage can be written as:

$$
\begin{aligned}
& D_{1}^{s}=y-p_{1}^{s}+\beta s_{s}^{s} \\
& D_{1}^{r}=y-p_{1}^{r}+\beta s_{r}^{r} \\
& D_{1}^{j}=y-p_{1}^{j}+\beta s_{r}^{j} .
\end{aligned}
$$

The market demand under three strategies in the spot sale stage is the same and is characterized as

$$
D_{2}^{i}=x-p_{2}^{i}+\theta s_{r}^{i}
$$

$x, y$ represent the basic market scale in the pre-sale and spot sale stage, respectively. For simplicity, the self-price coefficients in the pre-sale and spot sale stage are normalized to $1 . \beta$ and $\theta$ measure the 
marginal demand change with respect to the value-added service level in the pre-sale and spot sale stage, respectively. Considering the uncertainty caused by the waiting time of the pre-sale process, consumers in the pre-sale market pay more attention to the pre-sale service level than the spot scenario. Thus, we assume $0<\theta<\beta<1$.

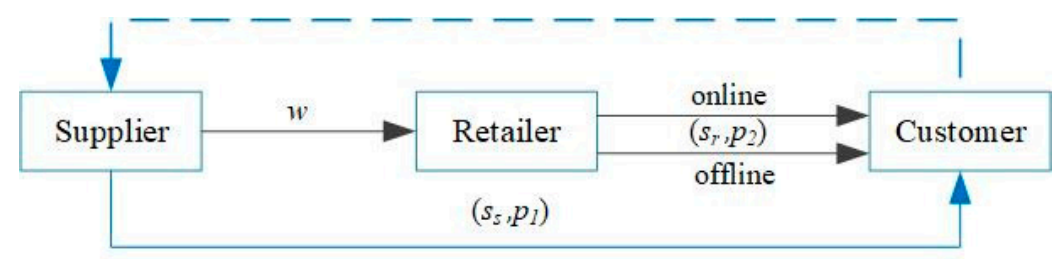

(a) Supplier pre-sale strategy

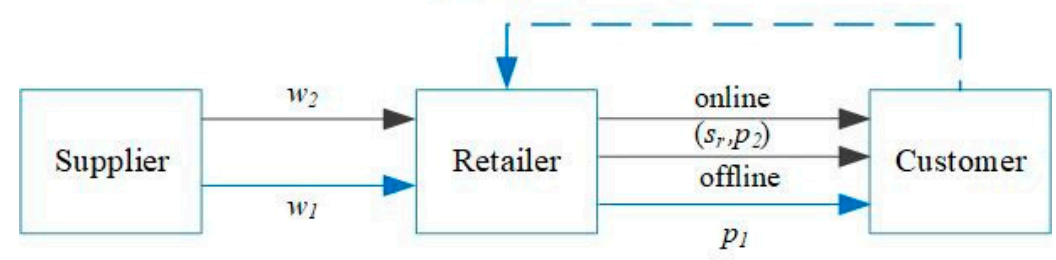

(b) Retailer pre-sale strategy

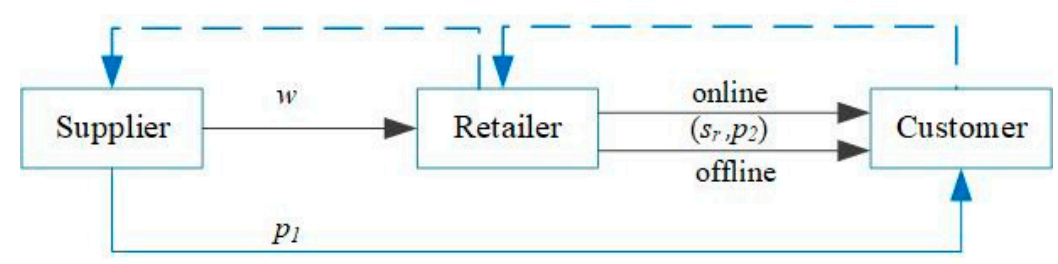

(c) Joint pre-sale strategy

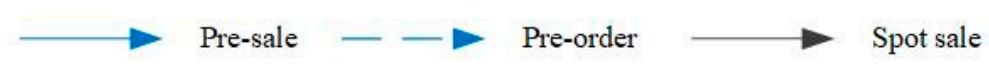

Figure 1. Structure of the fresh agri-product supply chain under three strategies: (a) Supplier pre-sale strategy, (b) Retailer pre-sale strategy (c) Joint pre-sale strategy.

We also provide some other assumptions as follows. (1) We assume that all information across the supply chain is common knowledge, and the retailer and the supplier are risk-neutral. Thus, the retailer and supplier make decisions to maximize their overall expected profits. (2) In a single sale cycle, the retailer and the supplier share information, and the retailer has no inventory left and does not consider the residual value of the product. (3) Under the retailer's pre-sale strategy, retailers do not distinguish between pre-sale and retail customers and provide the same level of service.

We summarize all notations in Table 1 , where $i(i=s, r, j)$ in superscripts represent the supplier pre-sale strategy, retailer pre-sale strategy, and joint pre-sale strategy, respectively.

\subsection{Supplier Pre-Sale Strategy}

In this scenario, the supplier provides the pre-sale service, such as cold-chain and customer service, whereas the retailer does not participate in pre-sale activities and still operates the spot sale. We consider this situation as the benchmark model. The supplier has to pay fixed costs to open a direct channel aside from the service cost due to the lack of a direct channel contacting the customer. In this Stackelberg game, the supplier initially decides the wholesale price, pre-sale price, and service level. Then, the retailer determines the spot sale service level and the sales price. 
Table 1. Notation.

\begin{tabular}{cl}
\hline Symbol & Definition \\
\hline Parameters & \\
$x$ & Basic market demand in spot sale market, $x>y$ \\
$y$ & Basic market demand in the pre-sale market \\
$c$ & Unit production cost \\
$\theta$ & The sensitivity of the market demand to the spot sale service level in the spot sale stage \\
$\beta$ & The sensitivity of the market demand to the pre-sale service level in the pre-sale stage \\
$a$ & Start-up costs for suppliers to open pre-sale channels \\
$m$ & Supplier's service improvement investment coefficient, $m>n$ \\
$n$ & Retailer's service improvement investment coefficient \\
$\lambda$ & The profit-sharing ratio from the supplier to the retailer \\
$D_{1}^{i}$ & The market demand in period 1 (pre-sale market) under three strategies \\
$D_{2}^{i}$ & The market demand in period 2 (spot market) under three strategies \\
$\pi_{s}^{i}, \pi_{r}^{i}$ & Profit of firms \\
\hline Decision variables \\
$p_{1}^{i}$ & Presale price, $p_{1}^{i}<p_{2}^{i}$ \\
$p_{2}^{i}$ & Spot sale price in the spot market \\
$w^{i}$ & Wholesale price, $p_{2}^{i}>w>c$ \\
$s_{r}^{i}$ & Retailer's service level \\
$s_{s}^{i}$ & Supplier's service level \\
\hline
\end{tabular}

Under the supplier pre-sale strategy, the profits of the supplier and the retailer can be expressed as:

$$
\begin{gathered}
\pi_{r}^{s}=D_{2}^{s}\left(p_{2}^{s}-w^{s}\right)-\frac{n s_{r}^{s 2}}{2} \\
\pi_{s}^{s}=D_{2}^{s}(w-c)+D_{1}^{s}\left(p_{1}-c\right)-a-\frac{m s_{s}^{s 2}}{2} .
\end{gathered}
$$

\subsection{Retailer Pre-Sale Strategy}

By contrast, under the retailer pre-sale strategy, the retailer presells products through his channels, whereas the supplier supplies wholesale pre-sale products to the retailer. Intuitively, adopting the retailer pre-sale strategy has some advantages. The supplier can reduce the cost of building and operating direct sales channels, and the retailer's online-to-offline mixed channel can also meet the operational requirements of pre-sale activities. Therefore, the supplier bears less risk in this scenario. On this basis, the supplier determines the wholesale price of pre-sale and spot sale products at $w_{1}^{r} w_{2^{\prime}}^{r}$ and then, the retailer sets the service level $s_{r}{ }^{r}$ and the sale prices $p_{1}^{r}$ and $p_{2}^{r}$.

Thus, we express the profits of the supplier and the retailer as:

$$
\begin{gathered}
\pi_{r}^{r}=\left(p_{2}^{r}-w_{2}^{r}\right) D_{2}^{r}+\left(p_{1}^{r}-w_{1}^{r}\right) D_{1}^{r}-\frac{n s_{r}^{r}}{2} \\
\pi_{s}^{r}=D_{2}^{r}\left(w_{2}^{r}-c\right)+\left(w_{1}^{r}-c\right) D_{1}^{r} .
\end{gathered}
$$

\subsection{Joint Pre-Sale Strategy}

Similar to the retailer pre-sale strategy, the suppliers also do not need to open a direct channel. The difference is that the suppliers view retailers as a pre-sale platform, and the retailer is responsible for selling and serving. In addition, the supplier establishes a revenue-sharing contract model to encourage the retailer to participate in the pre-sale cooperation alliance. Under this pre-sale strategy, the supplier sets the pre-sale and wholesale prices simultaneously; then, the retailer decides the service level and the spot price. As for the profit-sharing ratio, we assume that it is exogenous and depends on the bargaining power within the gaming process and varies from 0 to 1. 
Therefore, the profit for each member can be expressed as:

$$
\begin{gathered}
\pi_{r}=\left(p_{2}-w\right) D_{2}^{r}+\lambda\left(p_{1}-c\right) D_{1}^{r}-\frac{n s_{r}^{2}}{2} \\
\pi_{s}=D_{2}^{r}(w-c)+(1-\lambda)\left(p_{1}-c\right) D_{1}^{r} .
\end{gathered}
$$

\section{Results and Analysis}

In this section, we discussed the equilibrium results under three pre-sale strategies to figure out the differences among them. We applied the Stackelberg game led by the supplier to process three models which is very common in supply chain management research. Considering these assumptions under three models, we derive some results. The solution processes and the proof of results are shown in Appendix A.

\subsection{Equilibrium Analysis under Supplier Pre-Sale Strategies}

Applying the backward induction, substituting Equations (1) and (4) into Equations (5) and (6) respectively, we can obtain the optimal decisions of the supply chain players, which are showed in Lemma 1.

Lemma 1. Under the supplier pre-sale strategy, if $2 m-\beta^{2}>0$ and $2 n-\theta^{2}>0$, then we have equilibrium solutions:

$$
s_{s}^{*}=\frac{\beta(y-c)}{2 m-\beta^{2}} s_{r}^{*}=\frac{\theta(x-c)}{2\left(2 n-\theta^{2}\right)}, p_{2}^{s *}=\frac{c\left(n-\theta^{2}\right)+x\left(3 n-\theta^{2}\right)}{2\left(2 n-\theta^{2}\right)}, p_{1}^{s^{*}}=\frac{m y+c\left(m-\beta^{2}\right)}{2 m-\beta^{2}}, w^{s *}=\frac{c+x}{2} .
$$

According to Lemma 1, a high market scale in two markets contributes to a high selling price and service level, which is different from the relationship between the market scale and service level in Yu and Xiao [18]. When the supplier applies the supplier pre-sale strategy, the pre-sale market has no effect on the optimal decision of the retailer. Meanwhile, the optimal wholesale price also has no relationship with the pre-sale market.

Corollary 1. (1) $\frac{\partial s_{s}{ }^{*}}{\partial \beta}>\frac{\partial p_{1}^{s *}}{\partial \beta}>0, \frac{\partial s_{r}^{*}}{\partial \theta}>\frac{\partial p_{2}^{s *}}{\partial \theta}>0 ;$ (2) $\frac{\partial s_{r}^{s *}}{\partial n}<\frac{\partial p_{2}^{s *}}{\partial n}<0, \frac{\partial p_{1}^{s *}}{\partial m}<0, \frac{\partial s_{s}^{*}}{\partial m}<0$.

Corollary 1 shows the impact of consumers' freshness sensitivities and the service improvement investment coefficient on optimal decisions. Corollary 1 (1) indicates that high values of $\beta$ and $\theta$ motivate the retailer to increase the service-improving investment to improve the service level. This result is similar to that of Yu and Xiao [18]. In return, sales prices are raised to compensate for the high investment costs. Interestingly, we found that the sensitivity of market demand to service level has a more significant impact on service levels than sales price in either the pre-sale or spot sale markets. Therefore, the high service coefficient benefits the improvement in the service level at the pre-sale and spot sale stages. Meanwhile, consumers have to pay for outstanding services at a high price. Hence, both players can benefit from improving consumer sensitivities to service levels in their channels. Corollary 1 (2) shows that the service level and sale price under the supplier pre-sale strategy at the pre-sale and spot sale stages both decrease with the service improvement investment coefficient. That makes sense: the increasing service improvement coefficient will increase the operating pressure of the supplier and the retailer, thereby reducing the room for service improvement. Compared with spot prices, service levels are more affected by the retailer's service improvement investment. This means that although consumers spend less money, the level of service they enjoy drops even more under this situation, which potentially hurts consumers. 


\subsection{Equilibrium Analysis under Retailer Pre-Sale Strategies}

Similarly, by substituting Equations (2) and (4) into Equations (7) and (8), we obtain the following lemma 2.

Lemma 2. Under the retailer pre-sale mode, the equilibrium solutions exist if $2 n>\beta^{2}+\theta^{2}$, where $w_{1}^{r_{*}^{* *}}=\frac{y+c}{2}, w_{2}^{r *}=\frac{x+c}{2}, p_{1}^{r^{*}}=\frac{\left(2 n-\theta^{2}\right)(c+3 y)+\beta(x-c) \theta-2(c+y) \beta^{2}}{8 n-4 \beta^{2}-4 \theta^{2}}, p_{2}^{r *}=\frac{2 x\left(\beta^{2}-2 n\right)+\beta \theta(c-y)+(c+x)\left(\beta^{2}+2 \theta^{2}-2 n\right)}{4\left(\beta^{2}+\theta^{2}-2 n\right)}$, $s_{r}^{r^{*}}=\frac{\beta(y-c)+\theta(x-c)}{2\left(2 n-\beta^{2}-\theta^{2}\right)}$.

Lemma 2 shows that the optimal prices during two sale stages, the wholesale price and service effort, exist and are unique under the retailer pre-sale scenario when $2 n>\beta^{2}+\theta^{2}$. We find that the effect of the market scale on the equilibrium decisions is similar to that under the supplier pre-sale strategy. Figuring out the relationships between equilibriums is easy through the results in Lemma 2, and $\beta, \theta$ are analogous to Corollary 1 . With an increase in the consumers' service sensitivity in the pre-sale and spot sale stages, the service level and the selling price also increase. $\frac{\partial p_{2}^{r *}}{\partial n}<\frac{\partial p_{1}^{r *}}{\partial n}<0$ shows that when the cost coefficient of the service improvement increases, the spot sale price reduction is greater than the pre-sale price.

Then, we propose Propositions 1 and 2 to compare the optimal decisions between the retailer pre-sale model and supplier pre-sale model.

Proposition 1. (1) $p_{1}^{r *}>p_{1}^{s *}$, (2) $p_{2}^{r *}>p_{2}^{s *}$, (3) $s_{r}^{r *}>s_{s}^{s *}$, (4) $s_{r}^{r *}>s_{r}^{s *},(5) w_{2}^{r *}=w^{s *}$.

Proposition 1 (1) shows that the pre-sale price under the retailer pre-sale strategy is higher, which means that consumers participating in the pre-sale need to pay additional money to enjoy a great service. Besides, according to Proposition 1 (2), the customers in the spot sale market also need to pay for great services because of assumption 4, which is very common in real life. As for service level, Proposition 1 (3) indicates that the optimal service level for pre-sale under the retailer pre-sale strategy is greater than that under the supplier pre-sale strategy. The consumers could have a great convenient service under the retailer pre-sale strategy. Furthermore, Proposition 1 (4) demonstrates that the service level of the spot sale stage has also improved compared with the supplier's pre-sale strategy. In view of the service level, consumers would prefer the retailer pre-sale strategy. From Proposition 1 (1) to (4), it is evident that the improvement in service level results from retailers participating in pre-sale activities and generating more income. Besides, Proposition 1 (5) shows that the wholesale price in the spot sale stage under two strategies is not changed. Combining Proposition 1 (4) and (5), it is easy to find that the marginal profits are improving when the retailer carries out a pre-sale.

Proposition 2. (1) $D_{2}^{r *}>D_{2}^{s *}$; (2) $\pi_{r}^{r *}>\pi_{r}^{s *}$; (3) under the retailer pre-sale strategy, $\pi_{s}^{r *}=2 \pi_{r}^{r *}$, whereas under the supplier pre-sale strategy, $\pi_{s}^{s *}<2 \pi_{r}^{s *}$ if $a \geq \frac{m(c-y)^{2}}{2\left(2 m-\beta^{2}\right)}$, then $\pi_{s}^{s *}>2 \pi_{r}^{s *}$ if $a<\frac{m(c-y)^{2}}{2\left(2 m-\beta^{2}\right)}$

Proposition 2 (1) shows that when applying the retailer pre-sale strategy, the spot sale market demand is increasing due to the higher service level. From a retailer's perspective, although the pre-sale strategy increases sale prices and adds its service cost, the retailer still has chances to gain additional profits as the demand increases. Proposition 2 (2) reveals that the retailer's profit under the retailer pre-sale strategy is higher than that under the benchmark model. That is, although faced with higher costs, the retailer would like to participate in the pre-sale supply chain. If the start-up costs for suppliers to open pre-sale channels is too high, then the supplier will choose the retailer pre-sale strategy. Proposition 2 (3) shows that the rate of the supplier's profit to the retailer's profit is fixed under the retailer pre-sale strategy, and notably, the rate is lower than that under the supplier pre-sale strategy when the start-up costs are at a low level. At this moment, the profit gap between the retailer and the supplier is narrowed. The relationship of profits between the supplier and the retailer under 
two scenarios indicates that the supplier always achieves a higher profit than the retailer. This result is in line with our conventional wisdom.

\subsection{Equilibrium Analysis under Retailer Pre-Sale Strategies}

We can obtain equilibrium solutions by substituting Equations (3) and (4) into Equations (9) and (10), respectively. The results are presented in Lemma 3.

Lemma 3. Under the joint pre-sale strategy, the equilibrium solutions exist if $2 n>\theta^{2}, 2 \beta^{2} \lambda+\theta^{2}-2 n<0$ and $4 n(\lambda-1)\left(2 \beta^{2} \lambda+\theta^{2}-2 n\right)-(2 \lambda-1)^{2} \theta^{2} \beta^{2}>0$

$$
\begin{aligned}
& s_{r}^{j^{*}}=\frac{(c-x) \theta\left[\beta^{2} \lambda(2 \lambda-1)-2 n(\lambda-1)\right]-\beta(c-y)(\lambda-1)\left[2 \lambda\left(2 n-\theta^{2}\right)+\theta^{2}\right]}{A} \\
& p_{1}^{j^{*}}=\frac{2 n\left(2 n-\theta^{2}\right)(\lambda-1)(c+y)+\left[8 \beta^{2} c \lambda(1-\lambda)+\beta(c-x) \theta\right] n+\beta^{2} c(2 \lambda-1)^{2} \theta^{2}}{-A} \\
& p_{2}^{j^{*}}=\frac{\beta \theta(1-\lambda)\left[2 \lambda\left(2 n-\theta^{2}\right)+\theta^{2}-n\right](c-y)-B(c+3 x)+C \theta^{2}}{-A} \\
& w^{j^{*}}=\frac{\beta \theta(2 \lambda-1)(1-\lambda)\left(2 n-\theta^{2}\right)(c-y)-D(c+x)-\theta^{2} \beta^{2}(\lambda c+3 \lambda x-x)}{-A},
\end{aligned}
$$

where

$$
\begin{aligned}
& A=4 n(\lambda-1)\left(2 \beta^{2} \lambda+\theta^{2}-2 n\right)-(2 \lambda-1)^{2} \theta^{2} \beta^{2}, B=\beta^{2} \lambda\left(2 \lambda n+\theta^{2}-2 n\right)-2 n^{2}(\lambda-1), \\
& C=\beta^{2} x+2(c+x)\left(\beta^{2} \lambda^{2}-\lambda n-n\right), D=2\left(2 n-\theta^{2}\right)\left(\beta^{2} \lambda^{2}+n-\lambda n\right)-4 \beta^{2} \lambda n .
\end{aligned}
$$

Proposition 3. (1) $p_{1}^{j *}>p_{1}^{s *}$, if $\max \left(\lambda_{p_{1}}^{j s}, 0\right)<\lambda<1$ and $p_{1}^{j *} \leq p_{1}^{s *}$ if $0<\lambda \leq \max \left(\lambda_{p_{1}}^{j s}, 0\right)$.

(2) $p_{2}^{j *}>p_{2}^{s^{*}}$, if $\frac{n-\theta^{2}}{2\left(2 n-\theta^{2}\right)}<\lambda<1$ and $p_{2}^{j *}<p_{2}^{s *}$ if $0<\lambda<\frac{n-\theta^{2}}{2\left(2 n-\theta^{2}\right)}$.

(3) $s_{r}^{j *}>s_{s}^{s *}$, if $\max \left(\lambda_{s}^{j s}, 0\right)<\lambda<1$ and $s_{r}^{j *} \leq s_{s}^{s *}$ if $0<\lambda \leq \max \left(\lambda_{s}^{j s}, 0\right)$.

(4) $s_{r}^{j *}>s_{r}^{s *}$.

Proposition 3 shows that the relationships of the pre-sale price, spot sale price, and pre-sale service level under the joint and supplier pre-sale strategies are similar, and they all depend on $\lambda$. When $\lambda$ is greater than a certain threshold, the sale price and service under the joint pre-sale mode are higher than those under the supplier pre-sale strategy. This shows that the higher the share ratio, the higher the price of fresh products. The game between the supplier and the retailer eventually made consumers pay more money. For pre-sale price and service, we can infer that satisfying the conditions $p_{1}^{j *} \leq p_{1}^{s *}$ and $s_{r}^{j *}>s_{s}^{s *}$ is difficult; thus, consumers cannot enjoy a great pre-sale service at a low price under a joint pre-sale strategy. Thus, when the sharing ratio is too high and there are no other value-added products, this situation will leave consumers with a bad impression and they won't pay for it. However, for a spot sale, the service level at the spot stage may be high, and when the condition $p_{2}^{j *}<p_{2}^{s *}$ is satisfied, customers in the spot market will spend less money while enjoying better service. So, they prefer the joint pre-sale strategy to the supplier pre-sale one under this circumstance.

Proposition 4. (1) $D_{2}^{j *}>D_{2}^{s *}$; (2) $\pi_{r}^{j *}>\pi_{r}^{s *}$.

Proposition 4 (1) indicates that the joint pre-sale strategy increases the spot sale demand compared with the supplier pre-sale strategy. Combining Proposition 3, the positive impact of increased service levels is greater than the negative effect of increased prices. That is, consumers are willing to pay more for excellent service, which increases demand at the spot sale stage. As for the profit of the retailer, Proposition 4 (1) reveals that the retailer obtains additional profit under the joint pre-sale strategy compared with the supplier pre-sale one. This result is easy to understand: participating in pre-sales always brings more revenue to retailers, although they will face more costs. 


\section{Discussion}

\subsection{Comparison Of Three Pre-Sale Strategies}

We compared the retailer's pre-sale strategy and the joint pre-sale strategy with the benchmark model, then we conduct the comparative analyses among three pre-sale strategies to discuss which pre-sale approach has the highest service level and supply chain profit and determine how firms select the optimal pre-sale strategy in the supply chain through integrating the spot sale and pre-sale supply chain. In addition, we conduct the comparison of Proposition 5 based on assumptions $\lambda_{p_{1}}^{j s}>0, \lambda_{s}^{j s}>0$.

Proposition 5. (1) For the pre-sale price, $\left\{\begin{array}{l}p_{1}^{r *}>p_{1}^{s *}>p_{1}^{j *} \text { if } \quad 0<\lambda<\lambda_{p_{1}}^{j s} \\ p_{1}^{r *}>p_{1}^{j *}>p_{1}^{s *} \text { if } \quad \lambda_{p_{1}}^{j s}<\lambda<\lambda_{p_{1}}^{j r} \\ p_{1}^{j *}>p_{1}^{r *}>p_{1}^{s *} \text { if } \quad \lambda_{p_{1}}^{j r}<\lambda<1\end{array}\right.$

(2) For the spot sale price, $\left\{\begin{array}{lll}p_{2}^{r *}>p_{2}^{s *}>p_{2}^{j *} & \text { if } & 0<\lambda<\frac{n-\theta^{2}}{2\left(2 n-\theta^{2}\right)} \\ p_{2}^{r *}>p_{2}^{j *}>p_{2}^{s *} & \text { if } & \frac{n-\theta^{2}}{2\left(2 n-\theta^{2}\right)}<\lambda<\frac{1}{2} \\ p_{2}^{j *}>p_{2}^{r *}>p_{2}^{s *} & \text { if } & \frac{1}{2}<\lambda<1\end{array}\right.$

(3) For the pre-sale service, if $\lambda_{s}^{j s}<\frac{1}{2}$, then $\begin{cases}s_{r}^{r *}>s_{s}^{s^{*}}>s_{r}^{j *} & \text { if } 0<\lambda<\lambda_{s}^{j s} \\ s_{r}^{j^{*}}>s_{r}^{j *}>s_{s}^{S^{*}} & \text { if } \quad \lambda_{s}^{j s}<\lambda<\frac{1}{2} \text {; otherwise, } \\ s_{r}^{j *}>s_{r}^{r *}>s_{s}^{s^{*}} & \text { if } \quad \frac{1}{2}<\lambda<1\end{cases}$ $\left\{\begin{array}{lll}s_{r}^{r *}>s_{s}^{s *}>s_{r}^{j *} & \text { if } & 0<\lambda<\lambda_{s}^{j s} \\ s_{r}^{j *}>s_{r}^{r *}>s_{s}^{S *} & \text { if } & \frac{1}{2}<\lambda<1\end{array}\right.$.
(4) $\left\{\begin{array}{lll}w^{j *}>w^{{ }^{*}}=w_{2}^{r *} & \text { if } & 0<\lambda<\frac{1}{2} \\ w^{\varsigma *}=w_{2}^{r *}>w^{j *} & \text { if } & \frac{1}{2}<\lambda<1\end{array}\right.$.

From Proposition 5, we find that the sales price and service levels at the spot and pre-sale stage both increase as the profit-sharing ratio increases. Proposition 5 (1) provides a comparison of the pre-sale price. Although the pre-sale price is set by the supplier under the supplier pre-sale or retailer pre-sale strategy, the pre-sale price under the joint pre-sale strategy is the lowest when the profits sharing ratio is at a low level. By contrast, when the supplier shares the profit with the retailer at a high level, consumers will pay a correspondingly high pre-sale price. Therefore, as the profit-sharing ratio increases, the pre-sale price under the joint pre-sale mode will rise to the highest among the three strategies. Proposition 5 (2) reveals that the spot sale price increases with the profit-sharing ratio under a joint pre-sale strategy, which is similar to the results in Proposition 5 (1). Proposition 5 (3) demonstrates that the relationship of the pre-sale service level under three models resembles Proposition 5 (2). When the retailer gains additional profits from the supplier, the retailer has the initiative to improve the pre-sale service levels with a few risks. Increasing the sale price is feasible due to the increased service level. This result also means that in terms of the service level, the retailer pre-sale strategy can motivate the retailer to improve service levels more than the other strategies. Furthermore, the retailer can provide the highest level of service under the joint pre-sale strategy only when the supplier shares more than one-half of the pre-sale profit. From Proposition 5 (4), the wholesale price at the spot sale stage under the joint pre-sale strategy is the lowest when sharing additional profit with the retailer by more than one half, thereby contributing to the improvement of service levels.

For suppliers, $50 \%$ is a key threshold for profit sharing. When the sharing ratio is higher than $50 \%$ under the joint pre-sale strategy, the supplier not only has to be divided by the retailer for most of the pre-sale profit, the wholesale price to the retailer is also the lowest. Next, we compare the supplier's optimal profits under three pre-sale strategies directly to determine which strategy the supplier will prefer. With the assumption of $\lambda_{\pi_{s 2}}^{j s}<1$ and $\lambda_{\pi_{s 2}}^{r j}<1$, we can conclude $\lambda_{\pi_{s 2}}^{j s}>\lambda_{\pi_{s 2}}^{r j}$, and then, we obtain the supplier's pre-sale strategy selection. 


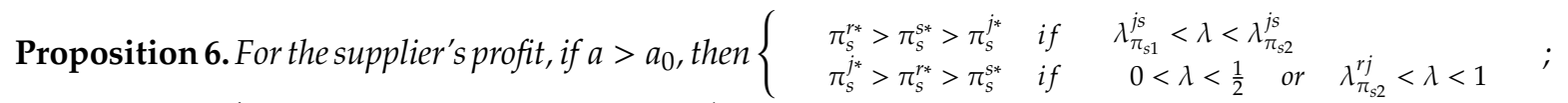
if $a<a_{0}$, then $\left\{\begin{array}{clcll}\pi_{s}^{s *}>\pi_{s}^{r *}>\pi_{s}^{j *} & \text { if } & \frac{1}{2}<\lambda<\lambda_{\pi_{s 2}}^{r j} & \\ \pi_{s}^{s *}>\pi_{s}^{j *}>\pi_{s}^{r *} & \text { if } & \lambda_{\pi_{s 1}}^{j s}<\lambda<\frac{1}{2} & \text { or } \quad \lambda_{\pi_{s 2}}^{r j}<\lambda<\lambda_{\pi_{s 2}}^{j s} \\ \pi_{s}^{j *}>\pi_{s}^{s *}>\pi_{s}^{r *} & \text { if } & 0<\lambda<\lambda_{\pi_{s 1}}^{j s} & \text { or } \quad \lambda_{\pi_{s 2}}^{j s}<\lambda<1\end{array}\right.$

Proposition 6 is provided to show the supplier's pre-sale strategy preference and it is shown graphically in Figure 2. SS, RS and JS in Figure 2 represent supplier pre-sale strategy, retailer pre-sale strategy and joint pre-sale strategy, respectively. The results show when $\lambda$ is relatively low or relatively high (Zone III in Figure 2), the supplier obtains the highest profit under the joint pre-sale strategy no matter how high the start-up capital (a). As revealed in Proposition 5, the low wholesale price reduces the supplier's profit gain from the spot sale stage, and the pre-sale price is the most economical. However, the low sale price stimulates consumer purchases and enables additional profits to the supplier than the other two strategies. When $\lambda$ is at a medium level, the relationship of the supplier's profits under three scenarios hinges on the initial start-up capital. Specifically, the supplier prefers to operate a preselling campaign by himself if much start-up capital is required (Zone I in Figure 2). Otherwise, cooperating with the retailer will be the first choice (Zone II in Figure 2). This result means that if the start-up capital is relatively high, the profit gained from the pre-sale campaign is not enough to cover the operating costs. Then, the retailer pre-sale strategy is the supplier's optimal selection. Furthermore, sharing additional profits with the retailer is difficult for the supplier. Therefore, the joint pre-sale strategy will be the dominant strategy for the supplier because of its wide-range applicability. Overall, our above analyses provide a logical mechanism for the supplier's pre-sale strategy selection in terms of start-up capital and optimal profit.

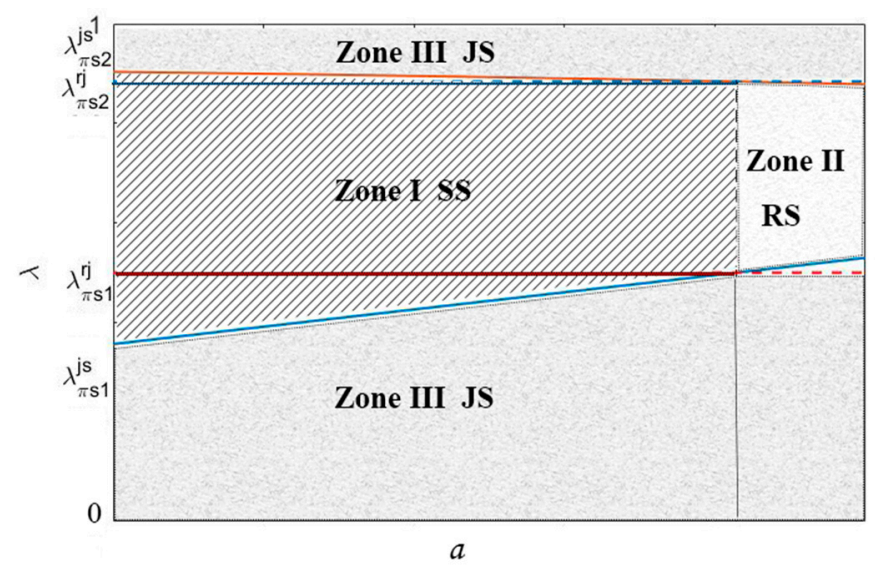

Figure 2. Pre-sale strategy selection preference of the supplier; SS, RS and JS represent supplier pre-sale strategy, retailer pre-sale strategy and joint pre-sale strategy, respectively.

Based on Propositions 2 (2) and 4 (2), we only need to compare the profits of the retailer under the under joint pre-sale and retailer pre-sale mode, and the relationship of the retailer's profits can be found, which also hinges on the profit-sharing ratio. Similarly, we assume that the threshold belongs to the interval $(0,1)$. Therefore, we can derive the following proposition.

Proposition 7. For the retailer's profit, $\left\{\begin{array}{lll}\pi_{r}^{r *}>\pi_{r}^{j *}>\pi_{r}^{S *} & \text { if } & 0<\lambda<\lambda_{\pi s}^{j r} \\ \pi_{r}^{j *}>\pi_{r}^{r *}>\pi_{r}^{s *} & \text { if } \quad \lambda_{\pi s}^{j r}<\lambda<1\end{array}\right.$.

Proposition 7 shows that the retailer's profit under supplier pre-sale models is always lower than the other two strategies. From the retailer's perspective, the retailer has stronger advantages than the supplier to carry out pre-sale activities; thus, when the retailer participates in pre-sales, the pre-sale project becomes a new profit growth point. Therefore, the retailer prefers to cooperate with 
the supplier to adopt the pre-sale. From Figure 3, notably, the preference hinges on the profit-sharing ratio. Specifically, when the profit-sharing ratio is higher than the threshold, the retailer obtains the highest profit under the joint pre-sale mode (Figure 3 Zone II). Considering Proposition 5 (2) and (4), as the spot sale price increases, the wholesale price decreases, thereby leading to a high marginal profit under the joint pre-sale strategy. Therefore, the retailer prefers the joint pre-sale strategy over the retailer pre-sale one. Under the joint pre-sale strategy, the increasing sharing ratio benefits the lower wholesale price and higher profits gained from the supplier, which enhances the advantages of the joint pre-sale strategy. On the contrary, when the sharing ratio is relatively low, the retailer always prefers the retailer pre-sale model rather than the joint pre-sale counterpart in this scenario.

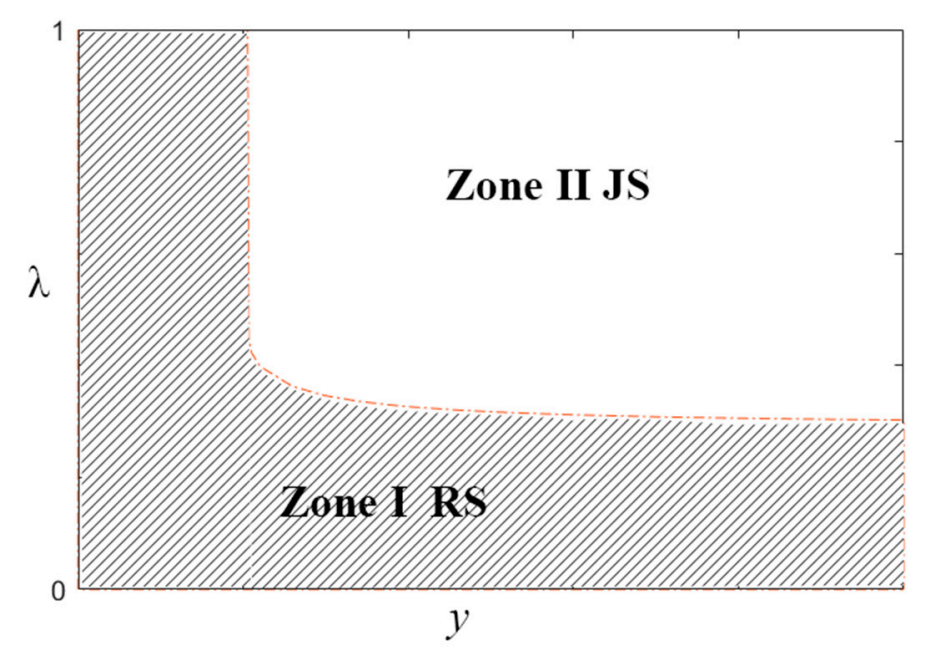

Figure 3. Pre-sale strategy selection preference of the retailer.

\subsection{Numerical Study}

Due to the complexity of the optimal solution formula, we also conduct numerical experiments in this section to verify the validity of the proposed models above and to explore how the basic pre-sale market size and profit-sharing ratio impact equilibrium decisions and profits. The default values of the parameters are used as follows: $x=500, y=200, c=0.2, a=250, \beta=0.6, \theta=0.4, m=1.2, n=1$. To meet the conditions $0<\lambda<1,2 \beta^{2} \lambda+\theta^{2}-2 n<0$, and $4 n(\lambda-1)\left(2 \beta^{2} \lambda+\theta^{2}-2 n\right)>(2 \lambda-1)^{2} \theta^{2} \beta^{2}$, we can calculate $0<\lambda<0.98$.

We explore the effect of pre-sale market size firstly. Figure 3 shows that the basic pre-sale market size plays an important role in determining the optimal pre-sale strategy of the supply chain members. In the first group of experiments, we evaluate how the pre-sale market size affects the equilibrium solution and profits. We assume that the profit-sharing ratio is fixed, we set $\lambda=0.4$, and the values of other parameters are the same as before.

Figure 4 depicts that from the consumers' perspective, if the pre-sale service level is high, then the price they pay is also high on the whole. Specifically, when the basic pre-sale market size is relatively low $\left(0<y<y_{1}\right)$, and the retailer pre-sale strategy is adopted, then consumers could enjoy the best service with a medium pre-sale price; if the joint pre-sale strategy is adopted, then consumers pay a high price for a medium service level. Figure $4 \mathrm{c}$ shows that the retailer strategy is the most sensitive to pre-sale market size among the three scenarios. Moreover, consumers in the spot sale market enjoy a great service if the retailer cooperates with the supplier to presell. Figure $4 b$,d also shows that the spot sale price is the highest under the retailer strategy, whereas the wholesale price is at a lower level, which incurs that the marginal profit at the spot sale stage is higher than other scenarios. Hence, the service rendered by the retailer is significantly better. That is, the retailer's unit profit gained from the spot sale market under the retailer pre-sale strategy is the highest, followed by the joint pre-sale strategy, and the lowest is the supplier pre-sale strategy. 


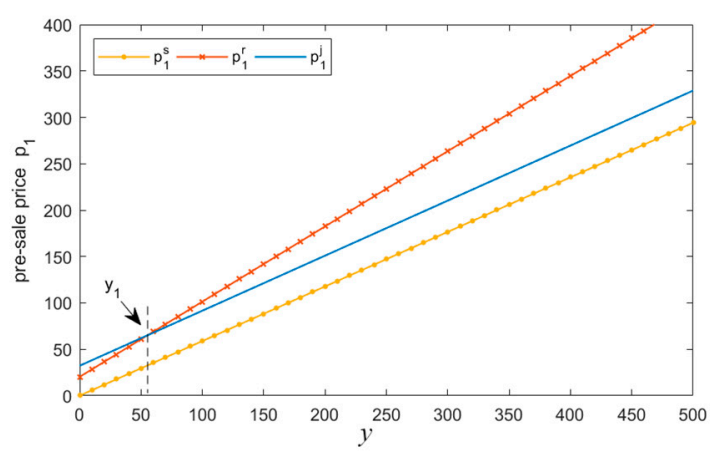

(a)

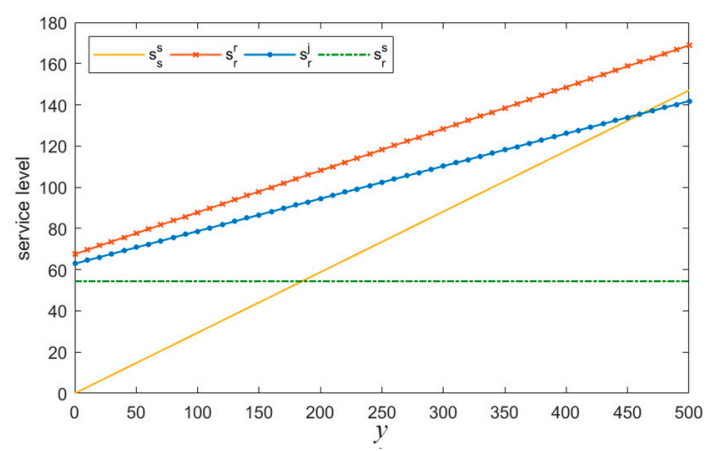

(c)

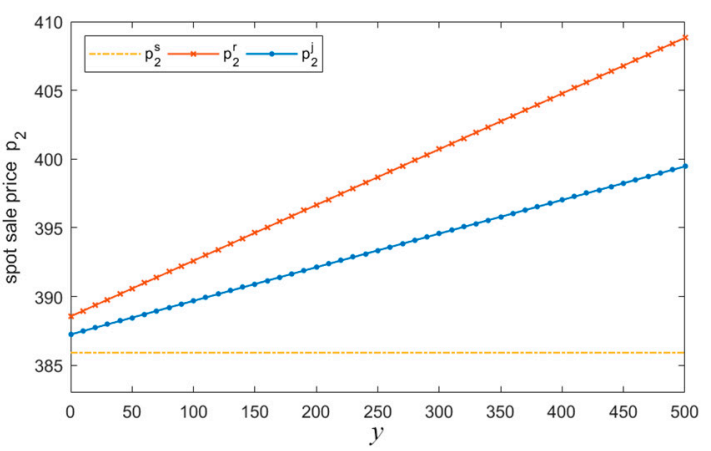

(b)

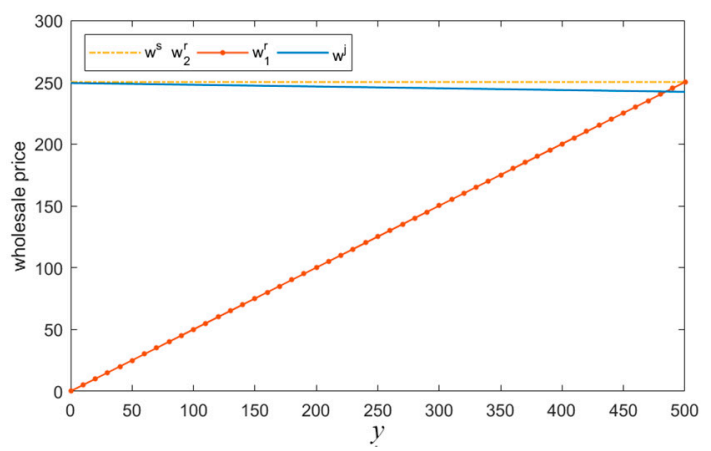

(d)

Figure 4. Optimal pre-sale price (a), spot sale price (b), service(c) and wholesale price (d) with respect to $y$ under the three strategies.

Figure 5 shows the impacts of the pre-sale market size on two players' profit. Comparing Figure $5 \mathrm{a}-\mathrm{c}$, it is obvious that the profits gap between the retailer and the supplier has narrowed as the profits sharing ratio increased under the joint pre-sale strategy. They also show us that when the sharing ratio is not very large, the profit gap between retailers and suppliers under the joint pre-sale strategy is gradually increasing. If the basic pre-sale market demand is not very large, then the supplier and the retailer hover between retailer pre-sale and joint pre-sale models. Given that both players have similar profits under the retailer or joint pre-sale strategy, the supplier has motivation to carry out the pre-sale by himself only when the basic pre-sale market size is extremely large. Comparing three scenarios when $\lambda=0.1,0.4,0.6$, we can find that the retailer and the supplier can gain high profit under the joint pre-sale strategy in most circumstances when the sharing ratio is moderate $(\lambda=0.4)$.

Then we discuss the effect of profit-sharing ratio. The profit-sharing ratio can change the profit of both members. Therefore, this ratio is a crucial influence factor of pre-sale strategy choices. In the first group of experiments, we evaluate the performance with different sharing fraction values. Figure 6 shows the results. 


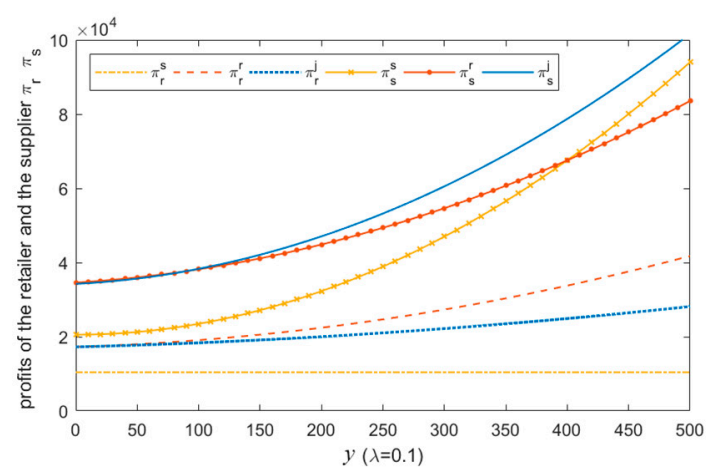

(a)

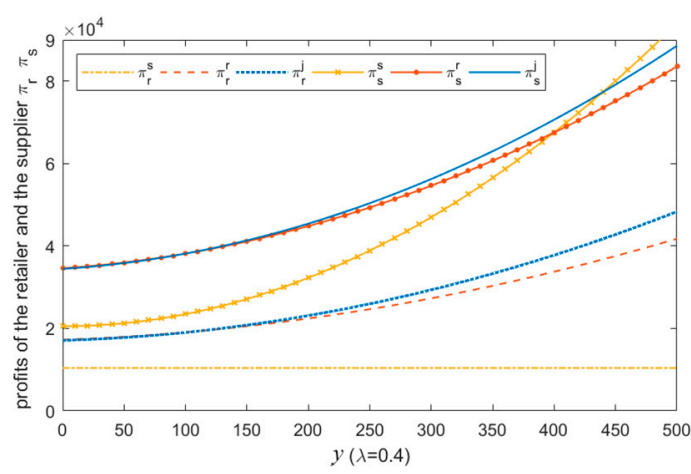

(b)

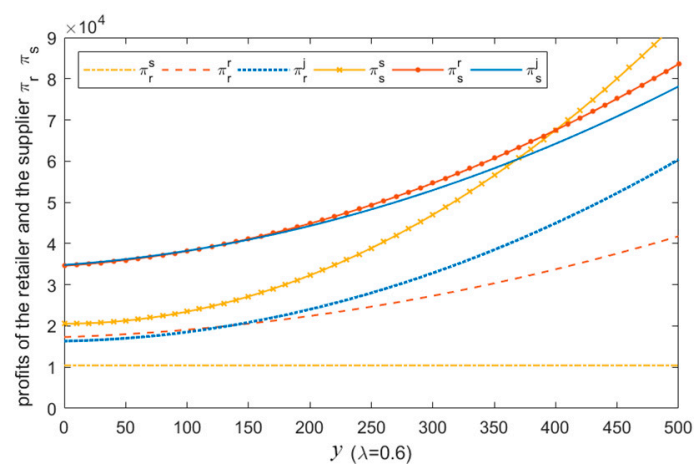

(c)

Figure 5. Profit of the retailer and supplier with respect to $y$ under three strategies when $\lambda=0.1$ (a), $\lambda=0.4(\mathbf{b})$ and $\lambda=0.6(\mathbf{c})$

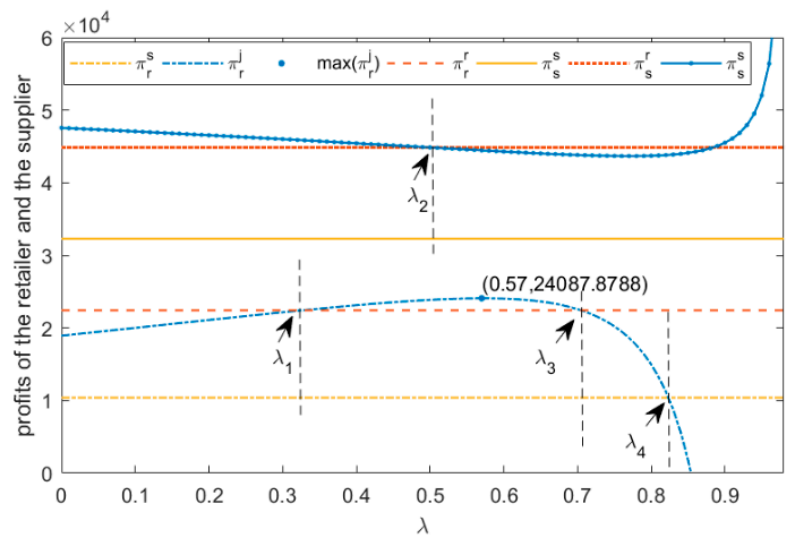

Figure 6. Profit of the retailer and supplier with respect to $\lambda$ under three strategies.

Figure 6 indicates that under the joint pre-sale strategy, the profit of the supplier increases initially and then decreases with $\lambda$, whereas the profit of the retailer would initially increase and then decrease along with the increasing sharing ratio. The increased profit shared by the supplier does not always benefit the retailer or negatively affect the supplier. This result might be contrary to our general understanding. However, considering Lemma 3, we could find the reason. The increased profit-sharing ratio has more impact on service levels than prices. The service level rises rapidly with the increase of sharing ratio, thereby resulting in increased service costs. On the one hand, when the sharing ratio is greater than a threshold, the profit of the retailer will decrease rapidly. On the other hand, the supplier can gain additional profit although $\lambda$ is increasing because of less cost and risk. 
Comparing the profits of the retailer and the supplier in three scenarios, we can obtain some thresholds. Then, we can draw some conclusions about the strategic choice of both parties. When the profit-sharing ratio is less than $\lambda_{1}$, the supplier pre-sale strategy is a dominating strategy for the supplier, whereas the retailer pre-sale strategy is highly attractive to the retailer. Then, when $\lambda_{1}<\lambda<\lambda_{2}$, the joint pre-sale strategy will be a common choice for all parties to achieve high profits. When $\lambda_{2}<\lambda<\lambda_{3}$, the supplier prefers the retailer pre-sale strategy, whereas the retailer would prefer the joint pre-sale strategy. This situation is just the opposite of that in $0<\lambda<\lambda_{1}$. If the sharing ratio continues to increase and $\lambda_{3}<\lambda<\lambda_{4}$, then the supplier and the retailer will make the same choice again. That is, both will prefer the retailer pre-sale strategy. If $\lambda_{4}<\lambda<1$, then the retailer will not participant in the pre-sale, which means that the supplier has to adapt to the supplier pre-sale strategy. However, the profit of the supplier is the lowest among the three scenarios. Hence, both parties will endeavor to negotiate for a great cooperation model. Figure 6 illustrates that when $\lambda_{1}<\lambda<\lambda_{2}$, the profit of both parties is higher than that when $\lambda_{3}<\lambda<\lambda_{4}$.

In summary, the retailer and the supplier will adopt the joint pre-sale strategy if $\lambda_{1}<\lambda<\lambda_{2}$, and negotiation is essential to decide the final selection. When the sharing ratio is zero, we notice that the retailer's profit under the joint strategy is still higher than that under the supplier pre-sale one. This notion at first seems to be inconsistent with common sense, but this case makes sense because of the low spot sale price and high service level.

In the previous analysis, we assume that the profit-sharing ratio is endogenous; now, we explore the case when the profit-sharing ratio is exogenous and determined by the retailer. Due to the complexity of these expressions, we plot the optimal sharing ratio under different scenarios in Figure 6, and we find that the retailer gains the highest profit when the supplier prefers the retailer pre-sale strategy. Then, we discuss the relationship between the optimal sharing ratio and parameters $\beta$ and $\theta$. With other parameter values remaining unchanged, $\beta$ and $\theta$ gradually change from 0 to 1 , and $\beta>\theta$. Figure 7 illustrates the relationship between retailer profits and profit-sharing ratio. In sum, the optimal sharing ratio decreases with $\beta$ and $\theta$. Therefore, if the supply chain applies the joint strategy and the retailer determines the profit-sharing fraction, then the retailer has the initiative to increase consumers' sensitivity to service level, such as the service differentiation strategy. If consumers' sensitivity to the service level is high, then the optimal sharing ratio is low, and it is easy to achieve this situation. Moreover, we calculated that the optimal profit-sharing rate varies from 0.23 to 0.99 . Combining the results shown in Proposition 6, we can roughly derive that if $0.23<\lambda<0.5$, then the retailer could gain more profit than in the situation shown in Figure 6.

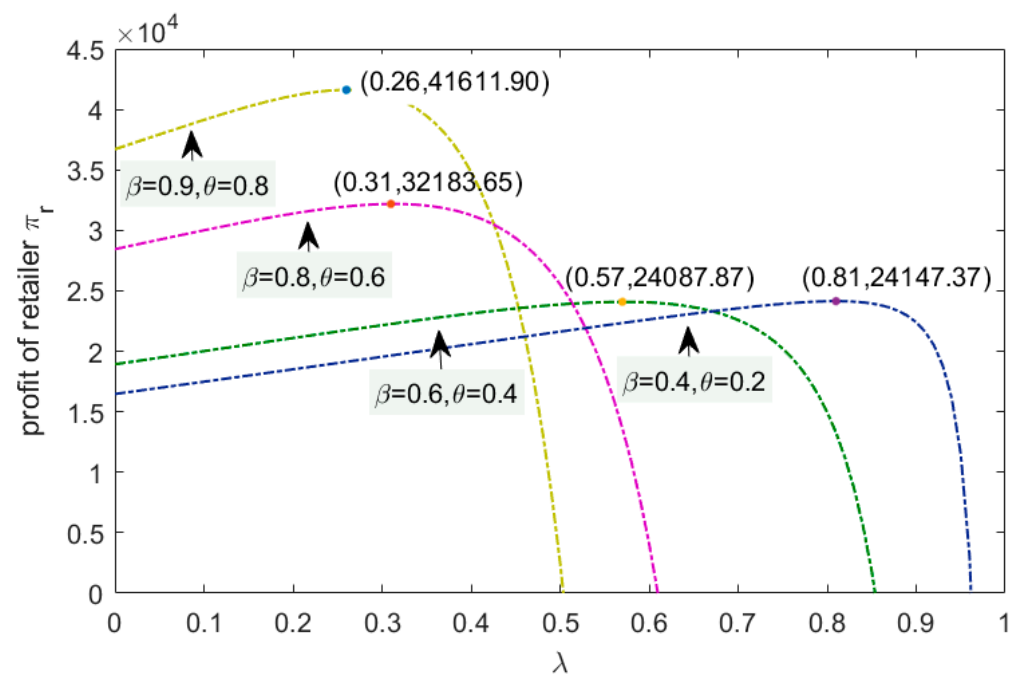

Figure 7. Optimal profit-sharing ratio with $\beta$ and $\theta$. 


\section{Conclusions}

In this study, we consider a supply chain consisting of a supplier who wants to adopt preselling to improve profits and a retailer who owns online and offline channels. Then, we introduce supplier pre-sale, retailer pre-sale, and joint pre-sale strategies into the supplier-led Stackelberg game. In order to find out the preference of supply chain players and their decision-making mechanism, we derive the optimal service level and pricing decisions in three pre-sale modes. Specifically, the optimal decisions are characterized in the paper, and numerical experiments are devised to evaluate the choice of pre-sale strategy.

Overall, we conclude our paper with the following main results: (1) The supplier pre-sale strategy is the lowest choice for the supplier because of the initial start-up capital and service costs, whereas the retailer and joint pre-sale will be the optimal choice for both parties. This result means that when the retailer and the supplier cooperate in carrying out preselling, both gain more profit than the supplier does alone. Moreover, no matter under which pre-sale strategy, the supplier always gains more profits than the retailer due to his leadership. (2) The basic pre-sale market demand and the profit-sharing ratio play an important role in the selection of two players. When the basic pre-sale market demand is not very large, then the supplier and the retailer hover between retailer pre-sale and joint pre-sale models, while when the sharing ratio is relatively low, the retailer pre-sale strategy will be the first choice of the retailer. Hence, they need to pay attention to consumer insight and negotiations on the profit-sharing ratio. Specifically, when the profit-sharing ratio is less than $50 \%$, both parties would likely cooperate by adopting the joint pre-sale strategy. (3) From the consumer's perspective, the principal of a highly senior service with a high price holds most of the time in three scenarios, but the service level improvement effect is more significant than the price increase effect due to the increasing pre-sale market demand and profit-sharing ratio. Thus, when consumers are willing to pay for better service, the three parties might achieve a multi-win scenario. (4) We also investigate the effect of the pre-sale market size and profit-sharing ratio and explored the extension scenario in which the profit-sharing ratio is endogenous under joint pre-sale strategies using numerical experiments. We found that the optimal profit-sharing ratio determined by the retailer is relevant to consumers' sensitivity to the service level. Therefore, the retailer has initiatives to improve service sensitivity in the spot sale and pre-sale stages. If the retailer increases customers' sensitivity to their service, then the retailer could easily achieve a higher profit.

This research can be extended in several directions in future work. We only consider the spot sale, and the pre-sale demand is assumed to be linear. However, the demand for fresh agri-products can be stochastic. Therefore, we can further consider the stochastic demand. In addition, we only considered the cooperation model and did not involve a competitive scenario. Thus, the competitive setting may be an interesting extension.

Author Contributions: H.Z. and X.P. conceptualized the study, supervised the data analysis; H.X. and H.Z. performed the data analysis and the results, methodology and contributed to the first draft. All authors have read and agreed to the published version of the manuscript.

Funding: This research was funded by the National Natural Science Foundation of China (Grant Numbers: 71871105).

Conflicts of Interest: The authors declare no conflict of interest.

\section{Appendix A}

Proof of Lemma 1. The supplier-led Stackelberg game can be analyzed by the backward induction procedure. We take the first-order derivation of $\pi_{r 2}^{s}$ with respect to $p_{2}^{s}$ and $s_{r}^{s}$, which is shown as follows:

$$
\frac{\partial \pi_{r 2}^{s}}{\partial p_{2}^{s}}=s_{r}^{s} \theta-2 p_{2}^{s}+w^{s}+x, \frac{\partial \pi_{r 2}^{s}}{\partial s_{r}^{s}}=\theta\left(p_{2}^{s}-w\right)-n s_{r}^{s} .
$$


Then, taking the second-order derivation, the Hessian matrix $H$ can be shown as: $H=\left[\begin{array}{cc}-2 & \theta \\ \theta & -n\end{array}\right]$; the Hessian matrix $H$ is negative definite if $2 n-\theta^{2}>0$. Therefore, $\pi_{r 2}^{s}$ is jointly concave in $p_{2}^{s}$ and $s_{r}^{s}$. $\frac{\partial \pi_{r 2}^{s}}{\partial p_{2}^{s}}=0$ and $\frac{\partial \pi_{r 2}^{s}}{\partial s_{r}^{s}}=0$, and we can have $p_{2}=\frac{w\left(n-\theta^{2}\right)+n x}{2 n-\theta^{2}}, s_{r}=\frac{\theta(x-w)}{2 n-\theta^{2}}$.

Subsequently, we solve the optimal decision of the supplier by substituting $p_{2}^{s}$ and $s_{r}^{s}$ into $\pi_{s 1}^{s}$. Then, we can obtain the Hessian matrix of $\pi_{s 1}^{s}$, where $\pi_{s 1}^{s}$ is the joint concavity on $p_{1}^{s}, s_{s}^{s}$, and $w^{s}$ when $2 m-\beta^{2}>0 . \frac{\partial \pi_{s 1}^{s}}{\partial p_{1}^{s}}, \frac{\partial \pi_{s 1}^{s}}{\partial s_{s}^{s}}$, and $\frac{\partial \pi_{s 1}^{s}}{\partial w^{s}}$ were set as zero, and then, the optimal decision of the supplier and the retailer can be obtained as shown in Lemma 1. Furthermore, we can have $\pi_{r}^{s *}=\frac{n(c-x)^{2}}{16 n-8 \theta^{2}}$, $\pi_{s}^{s *}=\frac{2 m n\left(3 c^{2}-2 c x-4 c y+x^{2}+2 y^{2}\right)-n \beta^{2}(c-x)^{2}-2 m \theta^{2}(c-y)^{2}}{4\left(2 n-\theta^{2}\right)\left(2 m-\beta^{2}\right)}-a, D_{2}^{s^{*}}=\frac{n(x-c)}{4 n-2 \theta^{2}}, D_{1}^{s^{*}}=\frac{m(y-c)}{2 m-\beta^{2}}$.

Proof of Corollary 1. According to Lemma 1 and the assumptions of $2 n-\theta^{2}>0,2 m-\beta^{2}>0$, we can have

$$
\begin{aligned}
& \frac{\partial p_{1}^{s *}}{\partial \beta}=\frac{2 \beta m(y-c)}{\left(\beta^{2}-2 m\right)^{2}}>0, \frac{\partial p_{1}^{s^{*}}}{\partial m}=\frac{\beta m(c-y)}{\left(\beta^{2}-2 m\right)^{2}}<0, \frac{\partial p_{2}^{*^{*}}}{\partial \theta}=\frac{\theta n(x-c)}{\left(2 n-\theta^{2}\right)^{2}}>0, \frac{\partial p_{2}^{s *}}{\partial n}=\frac{\theta^{2}(c-x)}{2\left(2 n-\theta^{2}\right)^{2}}<0, \\
& \frac{\partial s_{s}^{s^{*}}}{\partial \beta}=\frac{(y-c)\left(\beta^{2}+2 m\right)}{\left(\beta^{2}-2 m\right)^{2}}>0, \frac{\partial s_{s}^{s^{*}}}{\partial m}=\frac{2 \beta(c-y)}{\left(\beta^{2}-2 m\right)^{2}}>0, \frac{\partial s_{r}^{s^{*}}}{\partial \theta}=\frac{(x-c)\left(\theta^{2}+2 n\right)}{2\left(2 n-\theta^{2}\right)^{2}}>0, \frac{\partial s_{r}^{s^{*}}}{\partial n}=\frac{\theta(c-x)}{\left(2 n-\theta^{2}\right)^{2}}<0 .
\end{aligned}
$$

Proof of Lemma 2. Similar to the proof of Lemma 1, by substituting the optimal decisions into the supplier and retailer's profit functions, we have

$$
\begin{aligned}
& D_{1}^{r *}=\frac{(y-c)\left(2 n-\theta^{2}\right)+\beta \theta(x-c)}{4\left(2 n-\beta^{2}-\theta^{2}\right)}, D_{2}^{r *}=\frac{(x-c)\left(2 n-\beta^{2}\right)+\beta \theta(y-c)}{4\left(2 n-\beta^{2}-\theta^{2}\right)}, \\
& \pi_{s}^{r^{*}}=\frac{\left(4 n-\beta^{2}+2 \beta \theta-\theta^{2}\right) c^{2}+\left[2 \theta^{2} y-2 \beta(x+y) \theta+2 \beta^{2} x-4 n(x+y)\right] c-(\beta x-\theta y)^{2}+2 n\left(x^{2}+y^{2}\right)}{16 n-8 \beta^{2}-8 \theta^{2}} \\
& \pi_{r}^{r *}=\frac{\left(4 n-\beta^{2}+2 \beta \theta-\theta^{2}\right) c^{2}+\left[2 \theta^{2} y-2 \beta(x+y) \theta+2 \beta^{2} x-4 n(x+y)\right] c-(\beta x-\theta y)^{2}+2 n\left(x^{2}+y^{2}\right)}{32 n-16 \beta^{2}-16 \theta^{2}} .
\end{aligned}
$$

Proof of Proposition 1. Based on Lemma 1, we can derive the supplier and retailer's equilibrium profits under the supplier and retailer pre-sale strategies as follows:

$$
\begin{aligned}
& p_{1}^{r *}-p_{1}^{s^{*}}=\frac{(y-c)\left(2 \beta^{4}-3 \beta^{2} \theta^{2}+6 \beta^{2} n-2 m \theta^{2}-4 m n\right)+\beta \theta(x-c)\left(2 m-\beta^{2}\right)}{4\left(\beta^{2}+\theta^{2}-2 n\right)\left(\beta^{2}-2 m\right)}>0 \\
& p_{2}^{r *}-p_{2}^{s^{*}}=\frac{\beta \theta\left(\beta \theta(c-x)+(c-y)\left(2 n-\theta^{2}\right)\right)}{4\left(\beta^{2}+\theta^{2}-2 n\right)\left(2 n-\theta^{2}\right)}>0 ; \\
& s_{s}^{s^{*}}-s_{r}^{r *}=\frac{\theta(x-c)\left(\beta^{2}-2 m\right)+\beta(c-y)\left(\beta^{2}+2 \theta^{2}+2 m-4 n\right)}{2\left(\beta^{2}+\theta^{2}-2 n\right)\left(\beta^{2}-2 m\right)}<0 \\
& s_{r}^{r *}-s_{r}^{s^{*}}=\frac{\beta\left(\beta \theta(c-x)+(c-y)\left(2 n-\theta^{2}\right)\right)}{2\left(\beta^{2}+\theta^{2}-2 n\right)\left(2 n-\theta^{2}\right)}>0 .
\end{aligned}
$$

\section{Proof of Proposition 2.}

(1) $\quad D_{2}^{r}-D_{2}^{s}=\frac{\beta \theta\left[\beta(c-x) \theta+\left(2 n-\theta^{2}\right)(c-y)\right]}{4\left(\beta^{2}+\theta^{2}-2 n\right)\left(2 n-\theta^{2}\right)}>0$

(2) $\pi_{r}^{r}-\pi_{r}^{s}=\frac{(c-x)^{2} \beta^{4}-2 \theta(x-y)(c-x) \beta^{3}+E_{1} \beta^{2}+E_{2}-E_{3}}{16\left(2 n-\beta^{2}-\theta^{2}\right)\left(4 n-\beta^{2}-2 \beta \theta-\theta^{2}\right)}>0$

$E_{1}=\left(y^{2}+(2 c-4 x) y-2 c^{2}+2 x c+x^{2}\right) \theta^{2}+\left(-2 y^{2}+4 c y+4 x(c-x)\right) n$

$E_{2}=2\left((c-y) \theta^{2}-2 n(x-y)\right)(x-y) \theta \beta+(c-y)^{2} \theta^{4}$,

$E_{3}=2\left(2 x c-6 c y+3 y^{2}\right) n \theta^{2}+4\left(4 c y-x^{2}-2 y^{2}\right) n^{2}$ 
(3) $\pi_{s}^{r}=2 \pi_{r}^{r}$ and similarly, $\pi_{s}^{s}-2 \pi_{r}^{s}=\frac{m(c-y)^{2}}{2\left(2 m-\beta^{2}\right)}-a$, so when $a>\frac{m(c-y)^{2}}{2\left(2 m-\beta^{2}\right)}, \pi_{s}^{s}<2 \pi_{r}^{s}$, and when $a<\frac{m(c-y)^{2}}{2\left(2 m-\beta^{2}\right)}, \pi_{s}^{s}>2 \pi_{r}^{s}$.

Proof of Lemma 3. We obtain the first and second derivatives of $p_{2}^{j}$ and $s_{r}^{j}$ by applying the backward induction. Then, we set the first derivative to zero when the condition $2 n-\theta^{2}>0$ is satisfied to ensure the concavity; thus, we have $p_{2}^{j}=\frac{n(w+x)-\theta^{2} w-\beta \lambda\left(c-p_{1}\right) \theta}{2 n-\theta^{2}}, s_{r}^{j}=\frac{(x-w) \theta+2 \beta \lambda\left(p_{1}-c\right)}{2 n-\theta^{2}}$. Next, we obtain the profit of the supplier, that is, $\pi_{s}^{j}$ is a strictly concave function of $p_{2}^{j}$ and $s_{r}^{j}$ when $4 n(\lambda-1)\left(2 \beta^{2} \lambda+\theta^{2}-2 n\right)-\beta^{2} \theta^{2}(2 \lambda-1)^{2}>0$ and $2 \beta^{2} \lambda+\theta^{2}-2 n<0$. Then, we set the first derivatives of $w^{j}$ and $p_{1}^{j}$ equal to zero; thus, the optimal $w^{j}$ and $p_{1}^{j}$ can be obtained.

$$
\begin{gathered}
w^{j}=\frac{\left\{3 \beta(y-c) \theta^{3}-\left[(c+3 x) \beta^{2}+2 n(c+x)\right] \theta^{2}+6 n \beta(c-y) \theta+4 n\left(\beta^{2}+n\right)(c+x)\right\} \lambda+\beta(c-y) \theta^{3}}{+\left(x \beta^{2}+2 n(c+x)\right) \theta^{2}+2 n \beta(y-c) \theta-4 n^{2}(c+x)-2 \beta\left(2 n-\theta^{2}\right)[\beta(c+x)+\theta(c-y)] \lambda^{2}} \\
p_{1}^{j}=\frac{2(\lambda-1)(c+y) n\left(2 n-\theta^{2}\right)+\left[8 \beta^{2} c \lambda(1-\lambda)+\beta(c-x) \theta\right] n+\beta^{2} \lambda\left(\beta^{2} \theta^{2}\left(2 \lambda-\beta^{2}-n\right)+\left(\beta^{2}+4 n\right) \theta^{2}-8 n^{2}\right.}{4 n(1-\lambda)\left(2 \beta^{2} \lambda+\theta^{2}-2 n\right)+\beta^{2} \theta^{2}(2 \lambda-1)^{2}}
\end{gathered}
$$

We place $w^{j}, p_{1}^{j}, p_{2}^{j}$, and $s_{r}^{j}$ into $\pi_{r}^{j}$ and obtain the first derivative of $\lambda^{j}$ by combining $\frac{\partial \pi_{r}^{j}}{\partial \lambda^{j}}=0$ and $0<\lambda^{j}<1$; thus, $\lambda^{j}$ can be obtained.

Proof of Proposition 3. (1) $p_{1}^{j *}-p_{1}^{s *}=0$, considering that $0<\lambda<1$, we have one zero point $\lambda_{p_{1}}^{j s}$, and $0<\lambda_{p_{1}}^{\text {js }}<1$ is satisfied.

(2) $p_{2}^{j *}-p_{2}^{s *}=0$ has two roots, namely, $\lambda=\frac{n-\theta^{2}}{2\left(2 n-\theta^{2}\right)}$ and $\lambda=\frac{\beta(c-x) \theta}{2(c-y)\left(2 n-\theta^{2}\right)}+1$. As $0<\lambda<1$, we have $p_{2}^{j *}>p_{2}^{s *}$; if $\frac{n-\theta^{2}}{2\left(2 n-\theta^{2}\right)}<\lambda<1$ and $p_{2}^{j *}<p_{2}^{s *}$, then $0<\lambda<\frac{n-\theta^{2}}{2\left(2 n-\theta^{2}\right)}$.

(3) If $s_{r}^{j *}-s_{s}^{s *}=0$, then we have one zero point $\lambda_{s}^{j s}$, and the other is discarded for the condition $0<\lambda<1$.

(4) $s_{r}^{j^{*}}-s_{r}^{s^{*}}=-\frac{\left[2(\lambda-1)(c-y)\left(2 n-\theta^{2}\right)-\beta(c-x) \theta\right]\left[(1-2 \lambda) \theta^{2}+4 \lambda n\right] \beta}{2\left(2 n-\theta^{2}\right)\left\{\left[2 \beta^{2} \lambda^{2}-\left(\beta^{2}+2 n\right) \lambda+\beta^{2}+4 n\right] \theta^{2}+8 n(\lambda-1)\left(n-\beta^{2} \lambda\right)\right\}}>0$

Proof of Proposition 4. (1) $D_{2}^{j}-D_{2}^{s}=-\frac{\theta\left(2(1-\lambda)(y-c)\left(2 n-\theta^{2}\right)+\beta(x-c) \theta\right) n \beta}{2\left[\left(4(1-\lambda) n+(2 \lambda-1)^{2} \beta^{2}\right) \theta^{2}+8 n(\lambda-1)\left(n-\beta^{2} \lambda\right)\right]\left(2 n-\theta^{2}\right)}>0$; (2) Given that all the parameters and decision variables are positive, we have $M=\pi_{r}^{j *}-\pi_{r}^{s *}>0$. We omit the specific proof process here due to the complexity of the formula.

Proof of Proposition 5. $s_{r}^{j^{*}}-s_{r}^{r^{*}}=-\frac{(2 \lambda-1)\left\{(y-c)\left[2\left(2 n-\theta^{2}\right)^{2}(1-\lambda)+\beta^{2} \theta^{2}\right] \beta+\beta^{2}(c-x) \theta\left(2 \beta^{2} \lambda+\theta^{2}-4 n\right)\right\}}{2\left[4 n(\lambda-1)\left(2 \beta^{2} \lambda+\theta^{2}-2 n\right)-(2 \lambda-1)^{2} \theta^{2} \beta^{2}\right]\left(\beta^{2}+\theta^{2}-2 n\right)}, s_{r}^{j *}-s_{r}^{r^{*}}>0$ when $\frac{1}{2}<\lambda^{j}<1$, and $s_{r}^{j *}-s_{r}^{r *} \leq 0$ when $0<\lambda^{j} \leq \frac{1}{2}$.

$$
p_{2}^{j *}-p_{2}^{r *}=-\frac{\beta \theta(2 \lambda-1)\left\{\beta(c-x) \theta\left(2 \beta^{2} \lambda+\beta^{2}+2 \theta^{2}-6 n\right)+(y-c)\left[2\left(2 n-\theta^{2}\right)\left(\beta^{2}+2 \theta^{2}-4 n\right)(\lambda-1)+\beta^{2} \theta^{2}\right]\right\}}{4\left[4 n(\lambda-1)\left(2 \beta^{2} \lambda+\theta^{2}-2 n\right)-(2 \lambda-1)^{2} \theta^{2} \beta^{2}\right]\left(\beta^{2}+\theta^{2}-2 n\right)} ;
$$

that is, $p_{2}^{j *}>p_{2}^{r *}$ when $\frac{1}{2}<\lambda^{j}<1$, and $p_{2}^{j *}<p_{2}^{r *}$ when $0<\lambda^{j} \leq \frac{1}{2}$. Similarly, we can obtain the relationship between $p_{2}^{j *}, p_{2}^{\gamma^{* *}}$.

Combining the results we obtained in Propositions 3 and 4, Proposition 5 can be easily proved.

Proof of Proposition 6. $\pi_{s}^{r}>\pi_{s}^{s}$ when $a>a_{0}$; then, we compare $\pi_{s}^{j}, \pi_{s}^{s}$.

$$
\begin{aligned}
& \pi_{s}^{j}-\pi_{s}^{s}=0 \text { has two roots, namely, } \lambda_{\pi_{s 1}}^{j s} \text { and } \lambda_{\pi_{s 2}}^{j s} \text { thus, } \\
& \text { we have } \pi_{s}^{j}>\pi_{s}^{s} \text { if } 0<\lambda<\lambda_{\pi_{s 1}}^{j s} \text { or } \min \left(\lambda_{\pi_{s 2}}^{j s}, 1\right)<\lambda<1 \quad \text { and } \\
& \pi_{s}^{j}<\pi_{s}^{s} \quad \text { if } \quad \lambda_{\pi_{s 1}}^{j s}<\lambda<\min \left(\lambda_{\pi_{s 2}}^{j s}, 1\right) \text {. }
\end{aligned}
$$


$\pi_{s}^{r}-\pi_{s}^{j}=0$ has two roots, namely, $\lambda_{\pi_{s 1}}^{r j}=\frac{1}{2}$ and $\frac{1}{2}<\lambda_{\pi_{s 2}}^{r j} \min \left(\lambda_{\pi_{r 2}}^{j s}, 1\right)$; thus, we have $\pi_{s}^{r}<\pi_{s}^{j} \quad$ if $0<\lambda<\frac{1}{2} \quad$ or $\min \left(\lambda_{\pi_{s 2}}^{r j}, 1\right)<\lambda<1 \quad$ and $\pi_{s}^{r}>\pi_{s}^{j} \quad$ if $\frac{1}{2}<\lambda<\min \left(\lambda_{\pi_{s 2}}^{r j}, 1\right)$.

Considering $\lambda_{\pi_{s 2}}^{r j}>\lambda_{\pi_{s 2}}^{j s}$, Proposition 4 is proved.

Proof of Proposition 7. $\pi_{r}^{r *}>\pi_{r}^{s *}$ and $\pi_{r}^{j *}>\pi_{r}^{s *}$ are easily estimated. Then, $\pi_{r}^{j *}-\pi_{r}^{r *}=0$ is utilized to find the root $\lambda_{\pi r}^{j r}$. For simplicity, we assume $0<\lambda_{\pi r}^{j r}<1$; thus, Proposition 6 is proved.

\section{References}

1. Fay, S.; Xie, J.H. The Economics of Buyer Uncertainty: Advance Selling vs.Probabilistic Selling. Mark. Sci. 2010, 29, 1040-1057. [CrossRef]

2. Tang, C.S.; Rajaram, K.; Alptekinoğlu, A.; Ou, J. The Benefits of Advance Booking Discount Programs: Model and Analysis. Manag. Sci. 2004, 50, 465-478. [CrossRef]

3. Li, C.; Zhang, F. Advance Demand Information, Price Discrimination, and Preorder Strategies. Manuf. Serv. Oper. Manag. 2013, 15, 57-71. [CrossRef]

4. Xu, X.; Li, X.; Bian, Y.; Sun, Y. Advance selling in the presence of product diffusion effect. J. Syst. Sci. Syst. Eng. 2017, 26, 77-99. [CrossRef]

5. $\quad \mathrm{Li}, \mathrm{X}$; $\mathrm{Xu}, \mathrm{X}$.; Sun, Y. Advance selling strategies for oligopolists by considering product diffusion effect. Kybernetes 2016, 45, 744-759. [CrossRef]

6. Yang, S.; Shi, C.; Zhang, Y.; Zhu, J. Price competition for retailers with profit and revenue targets. Int. J. Prod. Econ. 2014, 154, 233-242. [CrossRef]

7. Fisher, M.; Raman, A. Reducing the Cost of Demand Uncertainty Through Accurate Response to Early Sales. Oper. Res. 1996, 44, 87-99. [CrossRef]

8. Shugan, S.M.; Xie, J. Advance-selling as a competitive marketing tool. Int. J. Res. Mark. 2005, 22, 351-373. [CrossRef]

9. Kevin Weng, Z.; Parlar, M. Integrating early sales with production decisions: Analysis and insights. IIE Trans. 1999, 31, 1051-1060. [CrossRef]

10. Boyac, T.; Özer, Ö. Information Acquisition for Capacity Planning via Pricing and Advance Selling: When to Stop and Act? Oper. Res. 2010, 58, 1328-1349. [CrossRef]

11. Guo, L. Service Cancellation and Competitive Refund Policy. Mark. Sci. 2009, 28, 901-917. [CrossRef]

12. Xiao, Y.B.; Zhang, J.H. Preselling to a retailer with cash flow shortage on the manufacturer. Omega 2018, 80, 43-57. [CrossRef]

13. Jin, W.; Luo, J.; Zhang, Q. Optimal ordering and financing decisions under advance selling and delayed payment for a capital-constrained supply chain. J. Oper. Res. Soc. 2018, 69, 1978-1993. [CrossRef]

14. Zhao, X.Y.; Stecke, K.E. Pre-Orders for New To-be-Released Products Considering Consumer Loss Aversion. Prod. Oper. Manag. 2010, 19, 198-215. [CrossRef]

15. Lim, W.S.; Tang, C.S. Advance Selling in the Presence of Speculators and Forward-Looking Consumers. Prod. Oper. Manag. 2013, 22, 571-587. [CrossRef]

16. Şeref, M.M.H.; Şeref, O.; Alptekinoğlu, A.; Erengüç, S.S. Advance selling to strategic consumers. Comput. Manag. Sci. 2016, 13, 597-626. [CrossRef]

17. Pi, Z.; Fang, W.; Zhang, B. Service and pricing strategies with competition and cooperation in a dual-channel supply chain with demand disruption. Comput. Ind. Eng. 2019, 138. [CrossRef]

18. Yu, Y.; Xiao, T. Pricing and cold-chain service level decisions in a fresh agri-products supply chain with logistics outsourcing. Comput. Ind. Eng. 2017, 111, 56-66. [CrossRef]

19. Luo, Y.; Nie, G.; Yuanyi, T. Service Decision with Consideration of Price in Competitive Market. In Proceedings of the Advances in Education and Management: International Symposium, Dalian, China, 6-7 August 2011; pp. 188-194.

20. Jin, M.; Li, G.; Cheng, T.C.E. Buy online and pick up in-store: Design of the service area. Eur. J. Oper. Res. 2018, 268, 613-623. [CrossRef] 
21. Xiao, T.; Yang, D. Price and service competition of supply chains with risk-averse retailers under demand uncertainty. Int. J. Prod. Econ. 2008, 114, 187-200. [CrossRef]

22. Wu, Q.; Mu, Y.; Feng, Y. Coordinating contracts for fresh product outsourcing logistics channels with power structures. Int. J. Prod. Econ. 2015, 160, 94-105. [CrossRef] 